XXVIII.

Aus der medicinischen Klinik der Universität Tokyo.

(Abtheilung des Prof. Dr. T. Aoyama.)

\title{
Klinische Beobachtungen über den Einfluss der Vagus- erregung auf das Auftreten heterotoper Herzreize.
}

Von

Dr. Ken Kuré.

(Hierzu T'afel XIII.)

Dass heterotope Herzreize bei der Erregung der Hemmungsnerven entstehen können, ist eine experimentell wohlbekannte Thatsache. Einschlägige klinische Beobachtungen sind bisher nur in geringer Zahl mitgetheilt worden. Ich hatte im vorigen Jahre bei Prof. Dr. T. A o yama in der medicinischen Klinik in Tokyo Gelegenheit, an zwei Fällen derartige Beobachtungen zu machen. Im ersten Falle handelt es sich um eine durch Vagustonussteigerung hervorgerufene Heterotopie, im zweiten Falle um automatische Kammerschläge während des Druckes auf den Vagus. Diese zwei Fälle zeigten ein typisches Verhalten. Ich glaube daher, dass diese beiden Fälle einer ausführlichen Mittheilung werth sind.

Ich fühle mich angenehm verpflichtet, an dieser Stelle meinem verehrten Lehrer Herrn Prof. Dr. T. Aoyaına für seine freundliche Leitung und Unterstützung bei dieser Arbeit meinen ergebensten Dank auszudrücken. Auch Herrn Dr. I. Yamakawa, unter dessen Behandlung die beiden angeführten Patienten standen, möchte ich hier für sein freundliches Entgegenkommen danken.

\section{Fall.}

Anamnese: 25jähriger Arbeiter, aufgenommen am 23. Mai 1910. Keinerlei hereditäre Belastung nachweisbar, im 14. und 21. Lebensjahr litt er angeblich an acutem Gelenkrheumatismus; seit dieser letzten Erkrankung klagt der Patient über Herzklopfen, Athembeschwerden, sowie heftige Kopfschmerzen.

Status praesens: Herzspitzenstoss: sichtbar und tastbar im V. Intercostalraum in der mittleren Axillarlinie, diffus verbreitert. Absolute Herzdämpfung: obere Grenze am unteren Rande der III. Rippe, rechte Grenze in der Medianlinie, linke Grenze in der mittleren Axillarlinie. Auscultation: An der Herzspitze ein diastolisches Geräusch, II. Pulmonalton accentuirt. Radialpuls: klein, mässig gespannt, unregelmässig, von normaler Frequenz.

Diagnose: Mitralstenose.

Am Abend des 28. Mai sank die Pulszahl plötzlich bis auf 30 in der Minute, in den folgenden drei Tagen wurde die Pulsfrequenz immer langsamer, dabei war der Puls immer sehr unregelmässig; während 
dieser Zeit habe ich mehrere Curvenaufnahmen gemacht. Die Auscultation des Herzens ergab keine Besonderheiten, nur war es sehr auffallend, dass das diastolische Geräusch sehr laut und abnorm lang war. Jeder Herzcontraction entsprach ein Radialpuls. Am 30. Mai injicirte ich $0,001 \mathrm{~g}$ Atropin. sulf. (frisch bereitet) subcutan, um den Angriffspunkt der die Frequenz herabsetzenden Ursache zu bestimmen. Vor der Atropininjection war der Puls, wie schon erwähnt, unregelmässig und langsam, 25 in der Minute, wie aus der Fig. 1 zu ersehen ist. 18 Minuten nach der Injection wurde der Puls ganz regelmässig, dabei betrug die Pulsfrequenz 50 pro Minute; nach 33 Minuten stieg die Pulsfrequenz bis zum Maximum, 76 in der Minute, wie Fig. 2 zeigt; nach zwei Stunden wurde der Puls wieder langsam und unregelmässig. Vor der Atropininjection machte ich auch wiederholt Vagusdruckversuche, dabei bemerkte ich eine abnorme starke Empfindlichkeit des Vagus. Durch doppelseitigen Vagusdruck ${ }^{1}$ ) bekam ich manchmal sehr langen Herzstillstand (53 Fünftelsec.). Fig. 3 zeigt einen 41 Fünftelsec. dauernden Stillstand des Pulses, hervorgerufen durch Vagusdruck; während dieses Pulsstillstandes sah ich keinen Venenpuls. Ausserdem ist zu bemerken, dass der liegende Patient während dieses Pulsstillstandes Schwindel- oder Unwohlgefühl nicht hatte. Ende Mai klagte der Patient über Brechneigung und Kopfschmerzen, die aber bald verschwanden. Am 1. Juni hatte der Patient einen sehr langsamen Puls, 19 in der Minute, und zwar merkwürdigerweise fast von regelmässiger Folge - trotzdem bisher stets starke Unregelmässigkeiten zu beobachten waren -, wie in Fig. $4 \mathrm{a}, 4 \mathrm{~b}$ und $4 \mathrm{c}$ zu sehen ist. Von diesem Tage an stieg die Pulsfrequenz wieder allmählich, am 7. Juni betrug die Pulszahl 70 in der Minute. Vom 7. bis 14. Juni schwankte die Pulsfrequenz zwischen 30 und 65 in der Minute, in den nächsten 5 Tagen schwankte sie um 35 in der Minute, dann kam wieder ein Stadium mit variabler Pulsfrequenz; dieser Zustand dauerte bis zu der am 29. Juni erfolgten Entlassung des Patienten an. Nach einem halben Jahre hatte ich Gelegenheit, diesen Patienten wieder zu untersuchen, dabei bemerkte ich wieder Symptome von Mitralstenose und etwas unregelmässigen Puls von normaler Frequenz; der Vagusdruck konnte jetzt aber nicht mehr einen so langen Herzstillstand hervorrufen.

\section{Analyse der Arterien- und Venenpulscurven.}

Fig. 1 wurde am 30. Mai aufgenommen, direct vor der Atropininjection; in der Curve sind nur der Venenpuls und der Radialpuls verzeichnet; ich möchte hier, bevor ich auf die Analyse der Curven eingehe, die Bemerkung vorausschicken, dass bei dieser Bradycardie die Zahl der Herzcontractionen immer gleich war der Pulszahl, was ich auscultatorisch und besonders durch zahlreiche gleichzeitige Aufnahme von Herzstoss und Arterienpuls festgestellt habe. Auf Grund dieser Controle ist es ohne Weiteres auszuschliessen, dass die langen Perioden

1) Mit Rücksicht auf die Erfahrungen Thanhoffor's (Centralbl. f. d. med. Wissensch. 1875. No. 25) habe ich bei gleichzeitigem Druck auf beide Vagi entsprechende Vorsicht angewandt. 
in dieser Curve etwa durch Extrasystolen verursacht waren. Die vollkommene Unregelmässigkeit der Pulsfolge spricht auch gegen Vorhofs- oder Ventrikelsystolenausfall. Nun kommt die Frage, ob diese Unregelmässigkeit zur Arhythmia perpetua gehört, weil es ja bekannt ist, dass die Arhythmia perpetua manchmal auch vorübergehend in regelmässige Pulsfolge übergehen kann. Gerade die Form der Unregelmässigkeit dieser Curve erinnert an Arhythmia perpetua. Die Venenpulscurve hat auch einige Aehnlichkeit mit der bei Arhythmia perpetua. Wenn man aber genauer ausmisst, so findet man, dass der Venenpuls eine Vorhofswelle zeigt und diese Vorhofswelle immer in demselben Intervall der Ventrikelcontraction vorausgeht, was man aus dem Vergleich mit dem Radialpuls schliessen kann; auch in den anderen Curven, die ich vor dieser Curve mit Venen- und Spitzenstossverzeichnung aufnahm, bemerkte ich ein regelmässiges Vorangehen der Vorhofswelle vor dem Spitzenstoss.

Also in diesem Venenpuls sieht man zwei Wellen, welche der Vorhofswelle und der Ventrikelstauungswelle entsprechen. Die Carotiszacke ist nicht bemerkbar. Dieses Vorhandensein einer Vorhofswelle schliesst eine Arhythmia perpetua aus; ebenso auch die folgende Thatsache: Um den Angriffspunkt der Ursache dieser Unregelmässigkeit zu bestimmen, habe ich $0,001 \mathrm{~g}$ Atropin subcutan gegeben; da wurde die Pulsfolge anfangs nur rascher, aber ohne dass die Arhythmie geschwunden wäre; als die Frequenz weiter die Höhe von 50 in der Minute erreichte, wurde der Puls ganz regelmässig.

Fig. 2 zeigt die Pulscurve, die auf dem Höhepunkt der Atropinwirkung aufgenommen ist, die Pulsfolge ist ganz regelmässig 76 in der Minute, jedem Radialpuls entspricht immer eine Vorhofswelle, durch Messung findet man, dass die Vorhofscontraction der Kammercontraction vorausgeht. Mit dem Abklingen der Atropinwirkung wurde der Puls wieder langsamer, dann plötzlich treten wieder längere Perioden auf, auch einzeln oder gruppenweise, aber das Längenverhältniss zwischen langen und kurzen Perioden zeigt keine Verdoppelung, was gegen Vorhofssystolenausfall spricht. Nach dem Resultate dieser Atropinwirkung muss man schliessen, dass die Ursache der Unregelmässigkeit mit einer Vaguswirkung in Zusammenhang steht.

Fig. 3 zeigt den Erfolg des Vagusdruckes; dieser Versuch wurde häufig vorgenommen, er hat immer einen auffallenden Herzstillstand hervorgerufen. Diese Curve, die vor Atropininjection während doppelseitigen Vagusdruckes aufgenommen wurde, zeigt einen 41 Fünftelsecunden dauernden Pulsstillstand; während desselben bemerkte man keinen Venenpuls, der eine Vorhofscontraction hätte verrathen können.

Fig. $4 \mathrm{a}$, Fig. $4 \mathrm{~b}$ und Fig. $4 \mathrm{c}$ wurden am 1. Juni aufgenommen, die Curve zeigte ein besonderes Verhältniss zwischen Vorhofs- und Kammercontraction, das mich zur Publication dieses Falles veranlasste. Wenn man Fig. 4a - an einer Stelle ist das Registrierpapier hängen geblieben, was man leicht aus der Zeitmarkirung ersehen kann - ansieht, so bemerkt man den im Vergleich mit Fig. 1 relativ regelmässigen Rhythmus der Ventrikelschläge. Im Venenpuls sieht man je einem 
Spitzenstoss entsprechend 2 Wellen; diese Venenwellen zeigen im Verlaufe der Curve einige Unterschiede; und zwar: die Venenpulse, die den ersten 5 Spitzenstössen entsprechen, zeigen eine andere Form als die folgenden Venenpulse. Die ersten 5 Venenpulse bestehen aus einer spitzigen schmalen und einer breiteren höheren Welle, dabei ist die Entfernung beider Erhebungen sehr klein; die folgenden Venenpulse sind zusammengesetzt aus einer breiteren, ersten Welle und einer etwas schmäleren, niedrigeren, zweiten Welle, dabei ist die Entfernung dieser beiden Erhebungen grösser als die entsprechende Entfernung bei den erstgenannten Venenpulsen.

Wenn man genauer ausmisst, so wird es klar, dass die zweite Erhebung aller Venenpulse der v-Welle entspricht. Bei den ersten 5 Venenpulsen entspricht die erste Erhebung, die spitzig aussieht und ein wenig später $(0,3-0,2$ Fünftelsecunde) als der Beginn der Kammercontraction sich erhebt, der c-Zacke (Carotiszacke); bei den übrigen Venenpulsen entspricht die erste Erhebung, die breiter aussieht, der Vorhofswelle + Carotiszacke, weil der Beginn dieser Erhebung um etwa 0,9 bis 0,6 Fünftelsecunden dem des Spitzenstosses vorangeht. Es ist daher klar, dass den Kammercontractionen bei den ersten 5 Schlägen keine Vorhofssystolen vorausgegangen sind, während bei den folgenden Schlägen Vorhofscontractionen porausgegangen waren. Warum die Ventrikelstauungswelle nach dem automatischen Kammerschlag früher auftritt und höher ist, kann man vielleicht in folgender Weise erklären: Entweder ist an ihrer Formation eine Vorhofswelle betheiligt, herrührend von einem rückläufig ausgelösten Vorhofsschlag, und diese Vorhofswelle täuscht ein frühzeitiges Eintreten der Ventrikelstauungswelle vor; oder bei Fehlen der Vorbofscontraction blieb der Vorhof im diastolischen $\mathrm{Zu}$ stand, und die vorhandene Staung gab Anlass zu dem frühzeitigen Eintritt der Ventrikelstauungswelle.

Um über diesen Punkt Aufschluss zu geben, möchte ich auf Fig. $4 \mathrm{~b}$ hinweisen, in dieser Curve sieht man auch zwei Arten von Venenpulsen. Die Venenpulse, die den ersten 7 Schlägen entsprechen, zeigen auch keine dem Carotispuls vorangehende a-Welle; die folgenden zwei Venenpulse haben eine deutliche a-Welle; vom nächsten Schlag an hat der Venenpuls wieder die Form wie bei den ersten 7 Schlägen. Der Beginn der $\mathrm{v}$-Welle der Venenpulse in dieser Curve ist bald ganz scharf, bald nicht ganz scharf, z. B. der Beginn der v-Welle des Venenpulses des 3., 4., 5., 6., 10. Schlages ist ganz scharf. Wenn man an diesen Stellen die Entfernung zwischen dem Beginn der c-Zacke und dem Beginn der v-Welle ausmisst, so ist die Entfernung sehr verschieden, z. B. das c- - -Intervall bei dem 6 . Schlage beträgt 1,7 Fünftelsecunde, das bei dem 10. Schlag beträgt 1 Fünftelsecunde. Wenn man dieser Thatsache Aufmerksamkeit schenkt, so wird es bald auffallen, dass die zweite Erklärung allein zur Erklärung des verschieden frühzeitigen Beginnes der v-Welle nicht genügt. Man muss hier vielmehr annehmen, dass der Vorhof nach der Kammer geschlagen hat. Die Thatsache, dass die Länge des $\mathrm{e}-\mathrm{v}$-Intervalles im Vergleich zu der normalen Ueberleitungs- 
zeit manchmal abnorm lang ist, spricht nicht gegen die Annahme, weil es auch möglich ist, dass die rückläufige Leitung in besonderen Fällen mehr Zeit als die normale Ueberleitung in Anspruch nimmt.

In Fig. $4 \mathrm{c}$ sieht man noch verschiedenere Formen des Venenpulses. Bei den zwei ersten Schlägen sieht man ein normales A-V-Intervall; bei dem dritten Schlag ist die Form des Venenpulses etwas anders, die erste Erhebung ist höher und breiter und von der zweiten Erhebung sieht man nur eine Andeutung; wenn man den Beginn der ersten Erhebung mit dem Beginn des Spitzenstosses vergleicht, so bemerkt man, dass sie sich fast gleichzeitig erheben, also es stellt sich der Beginn der ersten Erhebung früher ein als der Beginn der c-Zacke, die immer 0,2-0,3 Fünftelsecunde später als der Beginn des Spitzenstosses sich erhebt. So kann der Bcginn der ersten Erhebung des Venenpulses nicht durch die Kammercontraction hervorgerufen sein, sondern er wird durch die Vorhofscontraction verursacht sein. Also hier schlägt der Vorhof nur ein wenig früher als die Kammer. Bei der vierten Contraction fehlt die a-Welle vor der c-Zacke; der 5. Herzschlag ist von normalem a-v-Intervall; bei dem 6. ist die Schlagfolge der Vorhof- und Kammercontraction ganz wie bei dem 3. Schlage, nur ist die v-Welle hier deutlich zu sehen. Bei dem 7. Schlage ist der Venenpuls wieder etwas anders. Der Beginn der ersten Erhebung des Venenpulses erfolgt 0,3 Fünftelsecunde später als der Beginn des entsprechenden Spitzenstosses; die erste Erhebung des Venenpulses ist breit und hoch wie bei dem 3. Schlag. Man muss schliessen, dass der Beginn der Vorhofscontraction beim 7. Schlag nur ein wenig später als der der Kammercontraction erfolgt, die zweite Welle des diesem Herzschlage entsprechenden Venenpulses kommt nicht vorzeitig, während sie bei den übrigen Schlägen, bei denen die a-Welle im Venenpulse fehlt, immer mehr oder weniger vorzeitig sich einzustellen pflegt. Bei dem 8. Herzschlag fehlt wieder die a-Welle im Venenpuls; bei dem 9. schlägt der Vorhof nur ein wenig früher als die Kammer. In allen 3 Curven von Fig. 4 treten die Schläge, die von einem abnormen Venenpuls begleitet sind, immer vorzeitig im Vergleich zu dem Rhythmus der nor. malen Herzschläge auf.

\section{Fall.}

Anamnese: 50jähriger Kaufmann, aufgenommen am 18. Nov. 1909, entlassen am 11. Okt. 1910. Keinerlei hereditäre Belastung nachweisbar. Während der Kindheit war er ziemlich gesund, im 18. Lebensjahre inficirte er sich mit Syphilis. Im 28. Lebensjahre heirathete er eine gesunde Frau, bekam 5 Kinder, alle gesund; er ist ein mässiger Potator. Wegen Diabetes mellitus wurde er in unser Spital aufgenommen.

Status praesens: Herzspitzenstoss: sichtbar und tastbar im 5. Intercostalraum, direct ausserhalb der linken Mamillarlinie. Absolute Herzdämpfung: obere Grenze am oberen Rand der 3. Rippe, rechte Grenze am linken Sternalrand, linke Grenze in der linken Mamillarlinie. Auscultation: die Töne an allen Ostien ganz rein. Radialpuls: regelmässig, gut gespannt, Arterienwand etwas sklerotisch.

Dieser Mann klagte über keinerlei Beschwerden seitens des Herzens. Ich habe bei diesem Patienten zuerst, wie ich es gewöhnlich auszuüben 
pflegte, rechtsseitigen Vagusdruck gemacht, da habe ich sehr häufig eine besondere Allorhythmie bekommen, wie aus Fig. $5 \mathrm{zu}$ ersehen ist; solche Allorhythmie habe ich bei linksseitigem oder doppelseitigem Vagusdruck niemals wiederfinden können, wogegen ich dabei fast immer einfache Verlangsamung eventl. Herzstillstand bemerken konnte (s. Fig. 6).

\section{Analyse der Arterien- und Venenpulscurven.}

Die durch Vagusdruck ausgelöste Allorbythmie ist in Fig. 5 wiedergegeben. Wenn man die Curve näher ansieht, bemerkt man während der Wirkung des Vagusdruckes hintereinander abwechselnd lange und kurze Pulsperioden. Dieses Bild erinnert an Ventrikelsystolenausfall, der bei Vagusdruck nicht selten vorkommt. Die Besichtigung der Venencurve lehrt aber, dass die Allorhythmie in diesem Falle auf ganz andere Art zu Stande kommt.

Wenn man den Venenpuls, der dem nach einer längeren Pause auftretenden Radialpulse entspricht, ansieht, so bemerkt man, dass man hier nur eine $\mathrm{c}$-Zacke und eine $\mathrm{v}$-Welle findet, aber die a-Welle ganz vermisst. Dagegen sieht man an dem Venenpulse, der dem nach einer kürzeren Pause folgenden Radialpulse entspricht, ausser der c-Zacke und v-Welle noch eine Welle, die der c-Zacke in einem der normalen Ueberleitungszeit entsprechenden Intervall vorausgeht. Dass diese Welle nicht durch die vorausgehende Kammercontraction bedingt ist, kann man wohl dadurch ausschliessen, dass nach den vorzeitigen Kammerschlägen, denen eine längere Pause folgt, keine solche Erhebung auftritt; es kann sich also wohl nur um eine a-Welle handeln.

Also sehen wir, dass die Kammer ein Mal automatisch schlägt und das andere Mal sich auf einen vom Vorhof kommenden Reiz hin contrahirt. Es ist auffallend, dass die automatischen Kammerschläge in einem fast constanten Intervall den Contractionen mit normalem A-VIntervalle vorausgehen. Wenn man den Venenpuls genau ansieht, so wird man bald bemerken, dass die Carotiszacke, der keine a-Welle vorangegangen ist, im Vergleich zu der Carotiszacke, der eine a-Welle vorangegangen ist, auffallend hoch ist, und die der grossen c-Zacke folgende $\checkmark$-Welle immer auffallend grösser ist. Was die Vergrösserung der c-Zacke betrifft, so lässt sie sich vielleicht durch Stauung im Vorhof und in den Halsvenen in Folge des Ausbleibens der Vorhofscontraction erklären, weil bei einer solchen Stauung die vk-Welle besonders stark ausgebildet wird. Was das Anwachsen der $\nabla$-Wellen bei den heterotopen Schlägen betrifft, so kann man zu deren Erklärung ganz dieselben zwei Möglichkeiten, die ich für die Vergrösserung der v-Welle bei der Erklärung von Fig. 4 a hervorhob, anführen; dass es sich hier um eine rückläufige Vorhofcontraction handelt, möchte ich bezweifeln.

In Fig. 6 ist die Wirkung des linksseitigen Vagusdruckes wiedergegeben; man sieht nur eine Herabsetzung der Frequenz des Herzschlages. Doppelseitiger Vagusdruck brachte eine noch stärkere einfache Verlangsamung hervor; weil die Curve durch Unruhe des Patienten während des Vagusdruckes jedes Mal etwas verzerrt wurde, unterlasse ich es, diese Curve hier wiederzugeben. 


\section{Erörterung der Ergebnisse der Curvenanalyse beider Fälle.}

Bei der Erörterung der Ergebnisse der Curvenanalyse des ersten Falles hat man zunächst zu entscheiden, auf welche Weise die beschriebenen Aenderungen der Succession des Vorhofes und der Kammer zu Stande kommen. Es wäre daran zu denken, dass die verschiedene Grösse des Intervalles zwischen Vorhof und Kammer dadurch zu Stande kommt, dass bei unverändertem Vorhofrhythmus sich nur der Rhythmus der Kammer ändert, indem Kammersystolen von sehr geringerer Vorzeitigkeit auftreten. Wenn diese Erklärung auch für einzelne Stellen der Curven möglich erscheint, so lässt sie sich doch nicht durchweg durchführen, da die Rhythmusänderung an vielen Stellen sowohl den Vorhof wie die Kammer betrifft. Wir werden also annehmen müssen, dass wenigstens weitaus die meisten Herzschläge mit einem abnormen Intervall dadurch zu Stande kommen, dass der Reiz, der den Vorhof und die Kammer in Erregung versetzt, an einer abnormen Stelle entsteht. Da das Intervall zwischen Vorhofs- und Kammercontraction, bezw. Kammer- und Vorhofscontraction eine sehr verschiedene Dauer aufweist, so geht daraus hervor, dass der Ausgangspunkt des Reizes an sehr verschiedenen Stellen bei den verschiedenen Schlägen gelegen ist. An einzelnen Stellen ist das Intervall zwischen Vorhof und Kammer länger, als das Intervall zwischen Vorhof und Kammer bei der normalen Ueberleitung, ein Umstand, aus dem wir entnehmen müssen, dass der Ausgangspunkt des Reizes bei diesen Schlägen in die Kammer zu verlegen ist.

Es wäre nun die Frage aufzuwerfen, ob das Auftreten dieser Herzschläge mit verkürztem Intervall event. umgekehrter Succession in irgend einem Zusammenhang steht mit der gleichzeitig bestehenden starken Herabsetzung der Frequenz. Die Bradycardie war in unserem Falle auch vorhanden, bevor das Auftreten von Herzschlägen mit verkürztem Intervall event. mit umgekehrter Succession beobachtet wurde, sie verschwand nach Atropininjection, woraus hervorgeht, dass sie durch eine Steigerung des Vagustonus bedingt war. Nun ist aus experimentellen Untersuchungen bekannt, dass unter dem Einfluss einer Vaguserregung eine heterotope Automatie auftreten kann.

1904 beobachtete Lohmann am Schildkröten- und Kaninchenherz das Vorkommen atrioventriculärer Schläge während der Vagusreizung, und führte es auf eine Automatie der Brückenfasern zurück.

H. E. Hering beobachtete am Affenherzen das Auftreten von atrioventriculären Schlägen und automatischen Kammerschlägen bei Vagusreizung, welche Thatsache er folgender Weise erklärte: „Bei der Vagusreizung wäre die Ortsänderung der Ursprungsreize wohl so $\mathrm{zu}$ verstehen, dass diejenigen Stellen des Herzens, deren Automatio durch die Vagusreizung gerade am wenigsten beeinflusst wird, am leichtesten noch Ursprungsreize produciren können."

Ich habe auch in einer soeben erscheinenden Mittheilung Studien über atrioventriculäre Automatie publicirt; darin habe ich auch das Auftreten einer atrioventriculären Automatie in Folge Vagusreizung mitge- 
theilt. Die Curven, die ich gewonnen habe, haben mit den Curven des hier besprochenen klinischen Falles grosse Aehnlichkeit. Es scheint daher am wahrscheinlichsten, dass in unserem Falle die beschriebenen Herzschläge mit verkürztem Intervall event. umgekehrter Succession als eine heterotope Automatie aufzufassen sind. Die Verkürzung des A-VIntervalles, event. die umgekehrte Schlagfolge kann dadurch entstehen, dass die Reize sich abwechselnd an verschiedenen Stellen in der Gegend des T'awara'schen Knotens, event. in der Kammer bilden; wenn die Reizbildungsstelle mehr vorhofwärts gelegen ist, so schlägt der Vorhof früher als die Kammer, aber wenn die Reizbildungsstelle immer mehr kammerwärts zu liegen kommt, so erfolgt der Beginn der Kammercontraction immer früher im Vergleich mit dem Beginn der Vorhofscontraction, bis sich schliesslich rückläufige Schlagfolge einstellt. Dass die Wanderung der Ursprungsstelle des Herzreizes in diesem Falle mit der Respiration im Zusammenhang stehen konnte, ist nicht unwahrscheinlich, in dieser Hinsicht habe ich jedoch kein Beweismaterial, um etwas Sicheres auszusagen.

Was die Pathogenese der Vagusreizung betrifft, habe ich keinen sicheren Anhaltspunkt, es ist mir aber wahrscheinlich, dass es sich um eine Erregung im cerebralen Vaguscentrum handelte, weil der Patient am Ende Mai Brechneigung und Kopfschmerzen aufwies. Auch darauf, dass der Vagusdruck bei diesem Falle besonders starken Erfolg hatte, möchte ich besonders aufmerksam machen, weil J. Rihl auch bei seinem erwähnten zweiten Falle, dessen Zustandekommen er auf centrale Vagusreizung zurückführte, besonders deutliche Verlangsamung durch leichten Vagusdruck erzielen konnte.

Ich will noch beiläufig hier bemerken, dass dieser Patient bei langem Herzstillstand kein Schwindelgefühl hatte. Es ist bekannt, dass die Empfindlichkeit der einzelnen Menschen gegen Bradycardie verschieden jst und mit Veränderung der Hirnarterien im Zusammenhang steht. Eine Patientin mit vollständiger Dissociation, die ich lange Zeit beobachtete, hatte starkes Schwindelgefühl während der Bradycardie $(16-20$ in der Minute); aber in diesem Falle hatte der Patient kein Schwindelgefühl trotz der ebenso starken Bradycardie (19 in der Minute). Ja, er hatte selbst während eines in Folge Vagusdruckes auftretenden Herzstillstandes von 53 Fünftelsecunden keinerlei Schwindelgefühl.

J. Rihl schrieb über seinen zweiten Fall, dass das Auftreten einer hochgradigen, mehr oder minder lange Zeit andauernden Bradycardie unabhängig von einer Ueberleitungsstörung vom Vorhof zur Kammer lediglich in Folge eines frequenzhemmenden Einflusses des Vagus auf die Bildung der Ursprungsreize wohl nicht häufig zur Beobachtung gelangen dürfte.

Mein Fall zeigte eine ähnliche, noch hochgradigere Bradycardie von derselben Herkunft.

Was den zweiten Fall betrifft, so handelt es sich um eine heterotope Reizbildung, hervorgerufen durch künstliche Vagusreizung. Dass in diesem Falle die Kammer automatisch schlug, ist auf Grund der Analyse der Arterien- und Venenpulscurve zweifellos; die automatischen 
Kammerschläge folgten in einem fast constanten Intervall den vorangehenden Kammercontractionen; dieses Intervall entspricht gerade der Frequenz, die gewöhnlich bei Kammerautomatie beobachtet wird. Die Allorhythmie, die man in diesem Falle beobachtet, lässt drei Auffassungen $z$ u.

Erstens könnte die Allorhythmie auf folgende Weise entstehen: Die Kammerautomatie entstand während eines durch Vagusdruck bervorgerufenen Herzstillstandes; die automatische Kammercontraction hat rückläufig eine Vorhofsystole ausgelöst, der wieder eine Kammercontraction nach normalem A-V-Intervall folgt 1 ). Diese Auffassung ist deshalb wahrscheinlich, weil die Schläge mit normalem a-c-Intervall ganz regelmässig in einem bestimmten Intervalle den automatischen Kammerschlägen folgten. Andererseits scheint die Thatsache dagegen zu sprechen, dass das Intervall $z$ wischen der a-Welle und der vorangehenden c-Zacke ca. ${ }^{3 / 5}$ Secunden beträgt, welche im Vergleich mit dem in diesem Falle sonst zu beobachtenden a-c-Intervall sehr lang ist, aber man muss hier doch ins Auge fassen, dass, wie schon bei der Erklärung der Fig. 4 des ersten Falles erwähnt wurde, die Thatsache bekannt ist, dass die rückläufige Ueberleitungszeit länger als die normale rechtläufige Ueberleitungszeit sein kann.

Die zweite Auffassung der Entstehungsweise der Allorhytbmie ist folgende: Die Schläge mit einem normalen a-c-Intervall sind ganz unabhängig von den automatischen Kammerschlägen ausgelöst. Hier muss man noch bemerken, dass diese Schläge mit einem normalen a-cIntervall heterotop oder nomotop sein können. Im ersten Falle musste man also neben der Kammerautomatie noch eine auriculäre heterotope Automatie annehmen. Im zweiten Falle wären die Schläge mit normalem a-c-Intervall nicht von einer heterotopen, sondern von der nomotopen Reizbildungsstelle ausgelöst, d. h. die nomotope Reizbildung würde dann durch Vagusdruck stark verlangsamt, sodass die Kammern automatisch schlagen konnten. Die Reizbildung an der nomotopen Stelle wäre also nicht vollständig unterdrückt, sondern es bildeten sich in ihr mit sehr langsamem Rhythmus weiterhin Reize, die in Bezug auf die Kammerautomatie vorzeitige Herzschläge auslösten.

Nach dieser zweiten Auffassung muss man aber das Vorkommen eines Intervalles von constanter Grösse zwischen den automatischen Kammerschlägen und den Kammerschlägen mit dem normalen a-cIntervall als zufällige Erscheinung betrachten.

In dem hier beschriebenen Fall ist die Genese der Heterotopie von anderer Art als bei den bis jetzt publicirten Fällen von Kammerautomatie in Folge Vagusdruckes. Bei letzteren Fällen schlug die Kammer in Folge Reizleitungshemmung automatisch, während in diesem Falle die Kammerautomatie auf Hemmung der nomotopen Reizbildung durch Vagusdruck zurückzuführen ist.

1) Ein solcher Dreischlag $\mathrm{V}-\mathrm{A}-\mathrm{V}$ ist von H. E. Hering schon am Hnudoherzen beobachtet worden. Pflüger's Arch. 1907. Bd. 116. S. 143. Fig. 7. 
Eine selır interessante Thatsache habe ich bei Vagusdruck bei diesem Patienten beobachtet. Ich habe nämlich nur bei rechtsseitigem Vagusdruck die beschriebene Allorhythmie beobachtet, bei linksseitigem und doppelseitigem kam sie niemals vor. Diese Thatsache kann man vielleicht so auffassen: Wenn man den rechten Vagus allein reizt, so wurde vielleicht in diesem Falle nur die nomotope Reizbildung gehemmt, ohne dass die Reizbildung im automatischen Kammercentrum hemmend beeinflusst wurde. In Folge dessen schlug die Kammer automatisch während der langsamen nomotopen Reizbildung. Wenn man aber in diesem Falle beide Vagi oder nur den linken Vagus reizte, wurde die Reizbildung im automatischen Kammercentrum gleichzeitig gehemmt mit der nomotopen Reizbildung; deshalb bekam man nur eine einfache Bradykardie ohne Kammerautomatie. Diese Annahme stünde mit gewissen experimentellen Beobachtungen nicht im Widerspruch ${ }^{1}$ ). Es ist bemerkenswerth, dass dieser Patient niemals über irgend welche Beschwerden seitens des Herzens klagte; auch konnte ich bei Vagusdruck niemals Ventrikelsystolenausfall ausiösen. Ich möchte hier noch hinzufügen, dass diesem Kranken kein Digitalispräparat gegeben worden war.

\section{Besprechung der einschlägigen klinischen Literatur.}

J. Rihl hat in einer 1911 erschienenen Mittheilung ,Klinische Mittheilung über atrioventriculäre Automatie mit Bradykardie" zwei Fälle beschrieben, die mit meinem ersten Fall grosse Aehnlichkeit haben. Betreffs der Art und Weise, wic der Vagus das Auftreten ciner heterotopen Automatie fördert, hat er hervorgehoben, dass die Beobachtungr seines ersten Falles, bei-dem er fand, dass bei derselben Herzschlagfrequenz einmal atrioventriculäre Automatie, das andere Mal nomotope Automatie auftrat, beweist, dass das Auftreten der heterotopen Automatie eine gewisse Unabhängigkeit von der Höhe des Tonus der frequenzhemmenden Vagusfasern zeigt. Er hat diese Erscheinung folgenderweise erklärt: „Diese Erscheinung lässt sich wohl so verstchen, dass unter Einfluss des gesteigerten Vagustonus 1. verschiedene Stellen in der atrioventriculären Grenze eine nahezu ganz gleich hohe Automatic wie die normale Ausgangsstelle der Ursprungsreize erlangen, 2. die Reizbildung an der genannten Stelle nahezu vollständig synchron vor sich geht. Denn nur unter dieser Voraussetzung ist es erklärlich, dass ohne wesentliche Rhythmusänderung in Folge irgend welcher Umstände, die man sich als etwas raschere Reizbildung oder als geringfügigen Vorsprung in der Anspruchsfähigkeit vorstellen mag, bald die eine, bald die andere Stelle als Ausgangspunkt des den Herzschlag auslösenden Reizes fungirt."

Im Jahre 1909 hat Belski mitgetheilt, dass er im Verlaufe von Infectionskrankheiten mehr oder minder langdauernde atrioventrieuläre Automatie beobachtete, die von einer gewissen Bradykardie begleitet war;

1) Siehe auch die erst jüngst erschienene klinische Mittheilung ron Robinson, G. Canby und George Draper. Journ. of exp. med. 1912. Vol. 15. p. 14-37. 
und er hat hervorgehoben, dass die atrioventriculären Schläge in den betreffenden Fällen nicht verfrübt, sondern verspätet in Bezug auf den Rhythmus der normalen Schläge auftraten, wodurch er die extrasystolische Natur dieser Schläge ausgeschlossen hat. Sonst konnte or bei den betreffenden Patienten solche atrioventriculären Schläge manchmal durch Vagusdruck hervorrufen. Er hat aus seiner Beobachtung auch den Schluss gezogen, dass es sich um Vagustonussteigerung handelte.

Mackenzie ist der erste Autor, der eine Curve publicirte (1902), die das Vorkommen eines automatischen Kammerschlages deutlich zeigt. Eine 1908 von Joachim veröffentlichte Curve, die Joachim selbst in anderer Weise erklärt, zeigt, wie J. Rihl und Wenckebach auf Grund dieser Curve festgestellt haben, das Auftreten eines automatischen Kammerschlages während des Kammersystolenausfalles. Wenckebach hat auch in seiner Mittheilung 1908 eine Curve mit Kammerautomatie publicirt, die während eines Ventrikelsystolenausfalls entstanden war. Th. Lew is hat 1909 das Vorkommen automatischer Kammerschläge während einer periodischen Herabsetzung der Vorhofsfrequenz beobachtet. Die beiden letzten Autoren hatten die Erscheinung gleichfalls auf Steigerung des Vagustonus zurückgeführt.

Was das Auftreten automatischer Kammerschläge in Folge Vagusdruckes betrifft, hat J. Rihl in seiner Mittheilung 1908: "Klinischer Beitrag zur Kenntniss der Ueberleitungsstörungen von der Bildungsstätte der Ursprungsreize zum Vorhof" berichtet, dass die Kammerautomatie während einer Herabsetzung der Kammerfrequenz in Folge Vorhof- oder Ventrikelsystolenausfalles entstand. Und er hat hervorgehoben, dass die Digitalismedication die Kammerautomatie gesteigert haben kann, wie es im Thierexperiment v. Tabora nachgewiesen hat. In demselben Jahre hat Wenckebach in der oben erwähnten Mittheilung eine bei einem Vagusdruckversuch erhaltene Curve publicirt, die einen automatischen Kammerschlag während einer durch Ventrikelsystolenausfall bedingten Kammerpause zeigte. In meinem zweiten Fall war die Kammerautomatie nicht in Folge Ueberleitungsunterbrechung, sondern durch Hemmung der nomotopen Reizbildung in Folge Vagusdruckes entstanden.

\section{Zusammenfassung.}

In den vorliegenden zwei Fällen wurde durch Analyse gleichzeitig aufgenommener Arterienpuls- (bezw. Herzspitzenstoss-) und Venenpulscurven das Vorhandensein einer heterotopen Bradykardie festgestellt, die auf Vaguserregung bezogen werden muss. Im ersten Falle handelte es sich um eine ventriculäre und atrioventriculäre Bradykardie von etwa 20 Schlägen p. M. in Folge Steigerung des Vagustonus; nach Atropin trat eine Beschleunigung der Frequenz auf 76 Sehläge mit normaler A-V-Succession ein; durch Vagusdruck konnte Herzstillstand von über 10 Secunden hervorgerufen werden; während der Aenderung des Ausgangspunktes der Ursprungsreize schlug das Herz relativ rhythmisch. Im zweiten Falle traten in Folge rechtsseitigen Vagusdruckes Herzstillstand mit nachfolgender Kammerautomatie auf; während des Bestandes der 
Kammerautomatie folgte jedes Mal dem automatischen Kammerschlage in einem constanten Intervall ein Herzschlag von normalem a-c-Intervall in Form einer Extrasystole.

Am Schlusse meiner Abhandlung drängt es mich, Herrn Professor Dr. H. E. Hering für seine mannigfachen Aufklärungen bei der Analyse der Curven meinen ergebensten Dank auszusprechen.

\section{Litteratur.}

1. A. Belski, Beobachtungen über atrioventriculäre Automatie im Verlauf der Infectionskrankheiten. Zeitschr. f. klin. Med. Bd. 67. H. 5--6.

2. H. E. Hering, Experimentelle Untersuchungen über Herzuntersuchungen an Affen (1901). Diese Zeitschr. 1906. Bd. 2.

3. Joachim, Ein atypischer Fall von Störung der Reizleitung im Herzmuskel. Berliner klin. Wochenschr. 1908. No. 19. S. 911.

4. Th. Lewis, Irregular action of the heart in mitral stenosis: the inception of the ventricular rhythm. etc. Quarterly Journal of med. Vol.2. No.8. p.356. Juli 1909.

5. H. Lohmann, Zur Automatie der Brückenfasern und der Ventrikel des Herzens. Engelmann's Arch. 1904.

6. J. Mackenzie, The cause of heart irregularity in influenza. British medical journal. Nov. 1912.

7. J. Ribl, Klinischer Beitrag zur Kenntniss der Ueberleitungsstörungen von der Bildungsstätte der Ursprungsreize zum Vorhof. Deutsches Arch. f. klin. Med. 1908. Bd. 94.

8. Derselbe, Klinische Beobachtungen über atrioventriculäre Automatie mit Bradycardie.

9. K. F. Wenckebach, Beiträge zur Kenntniss der menschlichen Herzthätigkeit. Engelmann's Arch. Suppl. 1908.

\section{Erklärung der Figuren auf Tafel XIII.}

Zar Aufnahme der Curven warden Jaque t'scher Polygraph und Sphygmograph gebraucht. Die Zeit ist in Fünftelsecunden markiert.

Figur 1, 2, 3, 4a, 4b und $4 \mathrm{c}$ stammen vom Fall 1 , Figur 5 und 6 vom Fall 2. Figur 1,2 und 5 obere Curve $=$ Venenpuls, untere Curve $=$ Radialpuls.

Figur 3 und 6 Radialpuls.

Figur $4 \mathrm{a}, 4 \mathrm{~b}$ und $4 \mathrm{c}$ obere Curve $=$ Venenpuls, mittlere Curve $=$ Herzspitzenstoss, untere Curve $=$ Radialpuls.
$\mathbf{a}=$ Vorhofswelle
$\mathrm{V} . \mathrm{j} .=$ Vena jugularis
$\mathrm{c}=$ Carotiszacke
Cor $=$ Herzspitzenstoss
$\mathrm{v}=$ Ventrikelstauungswelle
A. $\mathrm{r} .=$ Arteria radialis. 


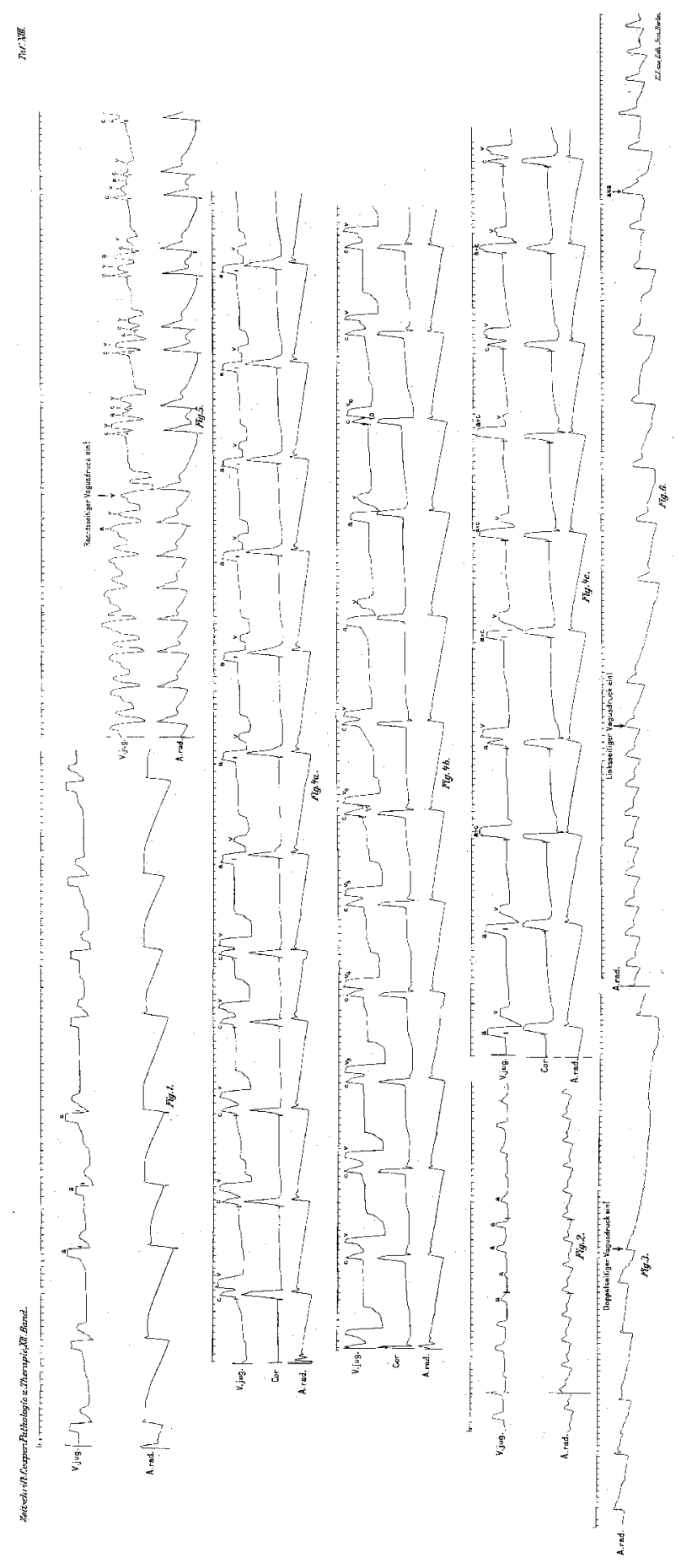

